\title{
A cost-effectiveness evaluation of the originator follitropin alpha compared to the biosimilars for assisted reproduction in Germany
}

This article was published in the following Dove Press journal: International Journal of Women's Health

\author{
Weiguang Xue' \\ Adam Lloyd' \\ Edel Falla \\ Claudia Roeder ${ }^{2}$ \\ Rudiger Papsch ${ }^{2}$ \\ Klaus Bühler ${ }^{3,4}$ \\ 'IQVIA, Real-World Evidence, London, \\ UK; ${ }^{2}$ Merck KGaA, Frankfurt, Germany; \\ ${ }^{3}$ Centre for Gynecology Endocrinology \& \\ Reproductive Medicine Kinderwunsch- \\ Zentrum Stuttgart, Stuttgart, Germany; \\ ${ }^{4}$ Department of Gynecology, Jena- \\ University Hospital-Friedrich Schiller \\ University, Jena, Germany
}

Background and objectives: Demand for assisted reproduction technology (ART) in Germany is high, with 100,844 treatment cycles during 2016. Many ART procedures involve ovarian stimulation with follicle stimulating hormone (FSH). Recently, biosimilar FSH products have become available. The objective of this study was to evaluate the costeffectiveness of the recombinant FSH Gonal- $\mathrm{f}^{(\mathbb{B})}$ (Originator) in comparison to biosimilar follitropin alfa, Bemfola ${ }^{\circledR}$ (Biosimilar 1) and Ovaleap ${ }^{\circledR}$ (Biosimilar 2), from a German payer perspective in terms of cost per live birth.

Methods: A decision tree model was developed, based on one cycle of assisted reproduction, to compare the original product to biosimilars. Clinical inputs, including live birth rates and adverse event rates were obtained from published randomized trials. Cost inputs were obtained from publicly available German sources. Clinical inputs, model structure and methodology were based on previous publications and validated by a clinical expert.

Results: Results indicated that the live birth rate is higher for the Originator compared to Biosimilar 1 (40.7\% vs 32.1\% respectively), and Biosimilar 2 (32.2\% vs $26.8 \%)$. The average cost per live birth for women treated with the Originator was estimated to be lower than those who were treated with biosimilars: Originator vs Biosimilar $1(€ 10,510$ vs $€ 12,192)$, Originator vs Biosimilar 2 ( $€ 12,590$ vs $€ 13,606)$. The analysis also found that the Originator is associated with an incremental cost-effectiveness of $€ 4,168$ and $€ 7,540$ per additional live birth versus Biosimilar 1 and Biosimilar 2 respectively. Sensitivity analysis indicated probabilities of pregnancy, embryo transfer and live birth, were key drivers of model costs. Scenario analysis confirmed the robustness of the model outcomes.

Conclusion: This study suggests that treatment with the Originator could result in a lower cost per live birth in comparison to biosimilars. Further analysis using real-world data, when available, is recommended to validate the results of the present study.

Keywords: reproductive techniques, follitropin alfa, cost per live birth
Correspondence: Edel Falla IQVIA, 210 Pentonville Road, London NI 9JY, UK

Tel +44203075 5794

Email edel.falla@iqvia.com

\section{Introduction}

Demand for assisted reproductive technology (ART) in Germany is high, with a reported 100,844 treatment cycles during 2016. ${ }^{1}$ This included 15,476 in-vitro fertilization (IVF) cycles, 50,111 intracytoplasmic sperm injection (ICSI) cycles and 24,842 cryo-transfer cycles. ${ }^{1}$ With restrictive public financing and a high cost burden, there is pressure to limit the costs of fertility treatments, whilst maximizing success rates. ${ }^{2,3}$ 
IVF and ICSI procedures both involve controlled ovarian stimulation (COS) with follicle stimulating hormone (FSH) injections. ${ }^{4}$ This strategy of stimulating ovaries, with gonadotropins is well established with the first generation of gonadotropins, produced from the urine of menopausal women, on the market since the 1970 's. ${ }^{5}$ Gonal- $\mathrm{f}^{\mathbb{B}}$, (Merck , Darmstadt, Germany; the Originator) is a fourth-generation gonadotropin, a recombinant FSH $(\mathrm{rFSH})$, which first entered the market in 1995, that has a well-established portfolio of published efficacy, safety and clinical real-world post-marketing evidence and experience. ${ }^{6-13}$

Follitropin alfa biosimilars (Bemfola ${ }^{\circledR}$, Gedeon Richter UK Ltd, London, UK [Biosimilar 1]; and Ovaleap ${ }^{\circledR}$; CVC Capital Partners, Luxembourg [Biosimilar 2]) have recently entered the market, with the sole purpose of producing cost savings over the originator product. Each biosimilar is supported by a single study powered to test non-inferiority in the number of oocytes collected compared with the originator product. ${ }^{14,15}$ Although gonadotropins are prescribed to stimulate the ovaries to increase egg production, the primary goal of administration is to achieve a live birth. ${ }^{16}$ By definition, a biosimilar medicine should be biologically similar to the reference medicine, with comparable safety and efficacy. ${ }^{17}$ However, differences in the batch-tobatch consistency between the products could lead to differences in effectiveness during stimulation cycles. For the Originator product, there is very low batch-to-batch variability $(<2 \%)$, ${ }^{18}$ which enables precise dosing. ${ }^{19}$ Indeed, the batch-to-batch variability for glycosylation profile and specific activity was assessed for $>200$ batches before the filled by mass and relative specific activity parameters were considered by the European Medicines Agency (EMA). However, the filled-by-mass calibration for biosimilars was assessed on only two pivotal clinical batches. Furthermore, a recent study comparing in-vivo biological activity and glycosylation between the Originator and Biosimilar 1 found differences in the glycan profile of the biosimilar, which may be associated with differences in receptor activation and biological activity (Biosimilar 1 potency was $14,522 \mathrm{IU} / \mathrm{mg}$ and the mean specific activity was $105.6 \%$ of the nominal value; the Originator potency was $13,159 \mathrm{IU} / \mathrm{mg}$ and the mean specific activity was $97.3 \%$ of the nominal value $[p=0.0048]) .{ }^{20}$ Although this was within the range stated in the product label, it clearly shows a difference between the two products.

Furthermore, while authorization of a biosimilar is based on similarity to the originator product, this does not necessarily imply that biosimilars are interchangeable, rather their use is regulated according to individual countries and the treating physician. ${ }^{21}$ Consequently, there is a pressing need to ensure that the choice of a biosimilar is fully informed, including any comparisons of costeffectiveness. As there are insufficient real-world data on which to base a suitably powered cost-effectiveness analysis on follitropin alfa biosimilars, the currently available multicenter randomized controlled trials that were conducted in broadly similar populations provide the most reliable data on pregnancy and live birth outcomes to date. ${ }^{14,15}$ As these trials were designed to enable marketing approval, we can assume that they reflect clinical use and, consequently, the data reported are robust enough to be used as the basis of the cost-effectiveness analysis. While this may not be standard methodology, there are precedents for this approach published in the literature. ${ }^{22-24}$

As the impact of biosimilars on costs per assisted reproduction treatment course and clinically meaningful outcomes has yet to be investigated in Germany, the objective of this study was to develop a costeffectiveness model to investigate the cost and clinical outcomes of the Originator in comparison to rFSH biosimilars from a German payer perspective, in terms of the cost per live birth.

\section{Methods}

Studies by Rettenbacher et al and Strowitzki et al, the two randomised controlled trials used to demonstrate similar efficacy and safety to the originator follitropin-alpha as part of the marketing authorization application of the biosimilar to the EMA, were chosen to inform clinical inputs for the comparison versus the Originator. ${ }^{14,15}$ These trials were chosen as no other studies for the Originator versus Biosimilar 1 or Biosimilar 2 where available at time of the analysis. These studies and the model we have employed have been used in several other estimates of the costeffectiveness of follitropin alfa. ${ }^{22-24}$

\section{Model structure}

A decision tree model was developed in Microsoft Excel 2013. The model was based on one cycle of assisted reproduction for the comparison of the Originator to both biosimilars (Figure 1). Key stages of one fresh cycle of assisted reproduction were defined in terms of eight discrete states (oocyte retrieval, no oocyte retrieval, embryo transfer, no embryo transfer, pregnancy, no pregnancy, live birth, and miscarriage). During the cycle, patients may also experience an adverse event from ovarian stimulation: ovarian hyper-stimulation syndrome 


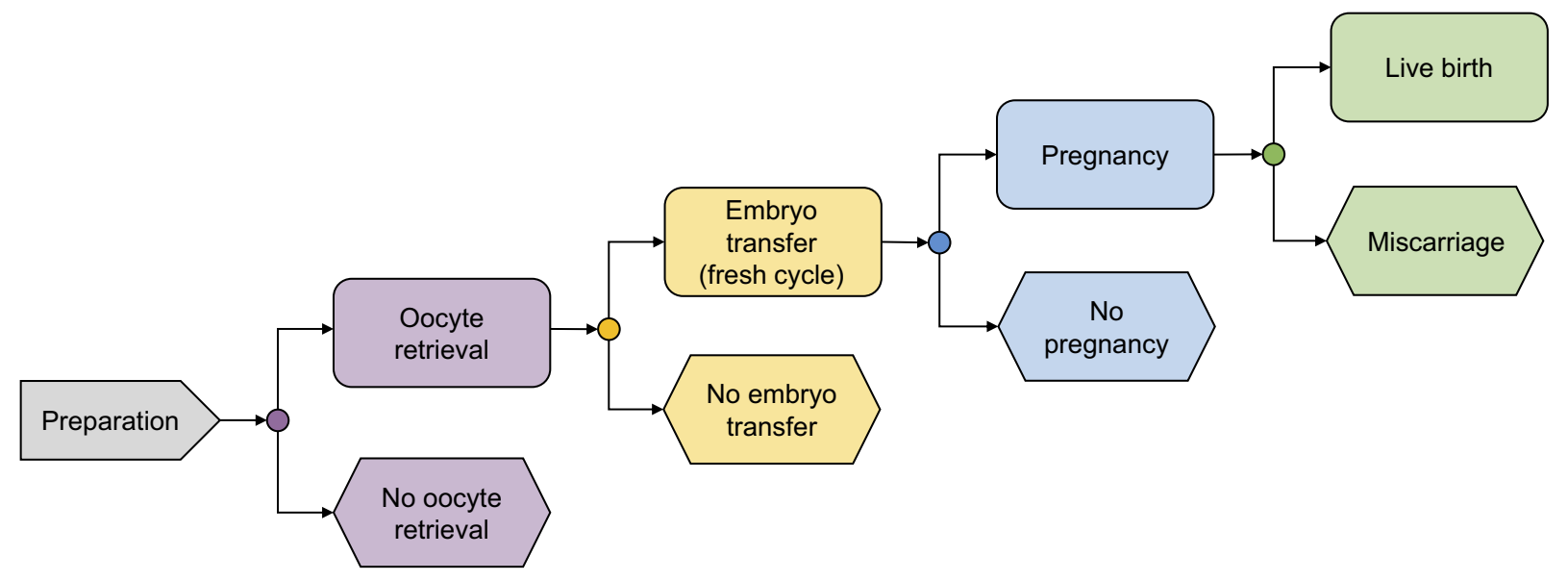

Figure I Cost-effectiveness model decision-tree structure.

(OHSS). The model was composed of four different pathway endpoints (no oocyte retrieval, no embryo transfer, live birth, no live birth).

Each of the model states was associated with a separate cost. The proportion of patients at the end of each treatment pathway, multiplied by the relevant cost and resource use, and the total sum of all pathways was used to generate overall costs for each intervention.

Model outputs included live birth rates, total costs, cost per live birth, and incremental cost-effectiveness ratio (ICER), estimated as the difference in costs divided by the difference in live birth rates of two comparators.

\section{Clinical inputs}

The probability of moving from one model state to another was based on the biosimilar clinical trial data (Rettenbacher et $\mathrm{al}^{14}$ and Strowitzki et $\mathrm{al}^{15}$ ). The primary objective of both Rettenbacher et $\mathrm{al}^{14}$ and Strowitzki et al ${ }^{15}$ was to demonstrate non-inferiority to the Originator, in terms of the number of oocytes retrieved. The rate of live birth was a secondary outcome in both trials.

The proportion of patients moving from oocyte retrieval to embryo transfer, etc were conditional on the success of the previous stage and, as such, the final birth rate, was conditional upon those who achieved an "ongoing pregnancy" (Table 1). "Ongoing pregnancy" rates $^{14,15}$ were extracted from trial data preferentially over "clinical pregnancy" rates.

Mild-moderate and severe OHSS rates were obtained from Rettenbacher et $\mathrm{al}^{14}$ and Strowitzki et $\mathrm{al}^{15}$ (Table 2). It was assumed that cycle discontinuation/interruption due to OHSS was captured in the "no oocyte retrieval" or "no embryo transfer" states.
A comparison between the Originator and both biosimilars together ("pooled biosimilars") was also conducted. A simple methodology was employed to estimate the pooled efficacy data, whereby the numbers of patients in the Originator, Biosimilar 1 and Biosimilar 2 arms of the Rettenbacher et $\mathrm{al}^{14}$ and Strowitzki et $\mathrm{al}^{15}$ trials, were combined to create a "pooled biosimilars" arm and pooled Originator arm. This enabled a combined analysis of clinical outcomes for the Originator versus both Biosimilar 1 and Biosimilar 2 (Table 1).

\section{Dosing}

Dosing was determined according to three levels of response to ovarian stimulation; normal, hyper and poor responders (Table 3). It was assumed that women who were "normal responders", defined as $>9$ and $\leq 15$ oocytes, received the mean dose of each intervention; "hyperresponders", defined as $>15$ oocytes, received the lower standard deviation of the mean dose; and "poor responders", defined as $<4$ oocytes received the upper standard deviation of the mean dose, as reported in each publication. ${ }^{25,26}$ For the pooled biosimilars analysis, dosing for Biosimilar 2, as reported in Strowitzki et al, was used as a proxy for "pooled biosimilars". 15 It was also assumed that the duration of stimulation (number of days) was equal to the mean duration of stimulation, for response type. The proportions for normal, hyper- and poor responders $(73 \%, 6 \%$ and $21 \%$, respectively) were used to estimate a weighted mean dose for each treatment. ${ }^{27}$

\section{Cost inputs}

Cost inputs were categorized into assisted reproduction and birth costs, adverse event costs and drug costs. 
Table I Transition probabilities

\begin{tabular}{|c|c|c|c|c|c|}
\hline \multicolumn{6}{|c|}{ Transition probability of oocyte retrieval } \\
\hline Analysis & Intervention & $\begin{array}{l}\text { Total number of } \\
\text { women in each arm }\end{array}$ & $\begin{array}{l}\text { Number of women } \\
\text { with oocyte } \\
\text { retrieval }\end{array}$ & $\begin{array}{l}\text { Probability of oocyte } \\
\text { retrieval }\end{array}$ & Reference \\
\hline \multirow{2}{*}{$\begin{array}{l}\text { Originator vs } \\
\text { Biosimilar I }\end{array}$} & Originator & 123 & 123 & $100 \%$ & \multirow{2}{*}{$\begin{array}{l}\text { Rettenbacher et al } \\
2015^{14}\end{array}$} \\
\hline & Biosimilar I & 249 & 249 & $100 \%$ & \\
\hline \multirow{2}{*}{$\begin{array}{l}\text { Originator vs } \\
\text { Biosimilar } 2\end{array}$} & Originator & 146 & 143 & $97.9 \%$ & \multirow{2}{*}{ Strowitzki et al $2016^{15}$} \\
\hline & Biosimilar 2 & 153 & 152 & $99.3 \%$ & \\
\hline \multirow{2}{*}{$\begin{array}{l}\text { Originator vs } \\
\text { pooled } \\
\text { biosimilars }\end{array}$} & Originator & 269 & 266 & $98.9 \%$ & \multirow{2}{*}{$\begin{array}{l}\text { Rettenbacher et al and } \\
\text { Strowitzki et al } 14,15\end{array}$} \\
\hline & $\begin{array}{l}\text { Pooled } \\
\text { biosimilars }\end{array}$ & 402 & 401 & $99.8 \%$ & \\
\hline \multicolumn{6}{|c|}{ Transition probability of embryo transfer } \\
\hline Analysis & Intervention & $\begin{array}{l}\text { Number of women } \\
\text { with successful oocyte } \\
\text { retrieval }\end{array}$ & $\begin{array}{l}\text { Number of women } \\
\text { that had embryo } \\
\text { transfer }\end{array}$ & $\begin{array}{l}\text { Probability of embryo } \\
\text { transfer conditional upon } \\
\text { oocyte retrieval }\end{array}$ & Reference \\
\hline \multirow{2}{*}{$\begin{array}{l}\text { Originator vs } \\
\text { Biosimilar I }\end{array}$} & Originator & 123 & 114 & $92.7 \%$ & \multirow{2}{*}{$\begin{array}{l}\text { Rettenbacher et al } \\
2015^{14}\end{array}$} \\
\hline & Biosimilar I & 249 & 224 & $90.0 \%$ & \\
\hline \multirow{2}{*}{$\begin{array}{l}\text { Originator vs } \\
\text { Biosimilar } 2\end{array}$} & Originator & 143 & 134 & $93.7 \%$ & \multirow{2}{*}{ Strowitzki et al $2016^{15}$} \\
\hline & Biosimilar 2 & 152 & $|4|$ & $92.8 \%$ & \\
\hline \multirow{2}{*}{$\begin{array}{l}\text { Originator vs } \\
\text { pooled } \\
\text { biosimilars }\end{array}$} & Originator & 266 & 248 & $93.2 \%$ & \multirow{2}{*}{$\begin{array}{l}\text { Rettenbacher et al and } \\
\text { Strowitzki et al } 14,15\end{array}$} \\
\hline & $\begin{array}{l}\text { Pooled } \\
\text { biosimilars }\end{array}$ & 401 & 365 & $91.0 \%$ & \\
\hline \multicolumn{6}{|c|}{ Transition probability of pregnancy } \\
\hline Analysis & Intervention & $\begin{array}{l}\text { Number of women } \\
\text { with embryo transfer }\end{array}$ & $\begin{array}{l}\text { Number of pregnant } \\
\text { women }\end{array}$ & $\begin{array}{l}\text { Probability of pregnancy } \\
\text { conditional upon embryo } \\
\text { transfer }\end{array}$ & Reference \\
\hline \multirow{2}{*}{$\begin{array}{l}\text { Originator vs } \\
\text { Biosimilar I }\end{array}$} & Originator & 114 & 51 & $44.7 \%$ & \multirow{2}{*}{$\begin{array}{l}\text { Rettenbacher et al } \\
2015^{14}\end{array}$} \\
\hline & Biosimilar I & 224 & 84 & $37.5 \%$ & \\
\hline \multirow{2}{*}{$\begin{array}{l}\text { Originator vs } \\
\text { Biosimilar } 2\end{array}$} & Originator & 134 & 49 & $36.6 \%$ & \multirow{2}{*}{ Strowitzki et al $2016^{15}$} \\
\hline & Biosimilar 2 & 141 & 42 & $29.8 \%$ & \\
\hline \multirow{2}{*}{$\begin{array}{l}\text { Originator vs } \\
\text { pooled } \\
\text { biosimilars }\end{array}$} & Originator & 248 & 100 & $40.3 \%$ & \multirow{2}{*}{$\begin{array}{l}\text { Rettenbacher et al and } \\
\text { Strowitzki et al }\end{array}$} \\
\hline & $\begin{array}{l}\text { Pooled } \\
\text { biosimilars }\end{array}$ & 365 & 126 & $34.5 \%$ & \\
\hline \multicolumn{6}{|c|}{ Transition probability of live birth } \\
\hline Analysis & Intervention & $\begin{array}{l}\text { Number of pregnant } \\
\text { women }\end{array}$ & $\begin{array}{l}\text { Number of women } \\
\text { with a live birth }\end{array}$ & $\begin{array}{l}\text { Probability of live birth } \\
\text { conditional upon } \\
\text { pregnancy }\end{array}$ & Reference \\
\hline \multirow{2}{*}{$\begin{array}{l}\text { Originator vs } \\
\text { Biosimilar I }\end{array}$} & Originator & 51 & 50 & $98.0 \%$ & \multirow{2}{*}{$\begin{array}{l}\text { Rettenbacher et al } \\
2015^{14}\end{array}$} \\
\hline & Biosimilar I & 84 & 80 & $95.2 \%$ & \\
\hline
\end{tabular}

(Continued) 
Table I (Continued).

\begin{tabular}{|l|l|l|l|l|l|}
\hline Analysis & Intervention & $\begin{array}{l}\text { Number of pregnant } \\
\text { women }\end{array}$ & $\begin{array}{l}\text { Number of women } \\
\text { with a live birth }\end{array}$ & $\begin{array}{l}\text { Probability of live birth } \\
\text { conditional upon } \\
\text { pregnancy }\end{array}$ & Reference \\
\hline $\begin{array}{l}\text { Originator vs } \\
\text { Biosimilar 2 }\end{array}$ & Originator & 49 & 47 & $95.9 \%$ & \multirow{2}{*}{ Strowitzki et al 2016 } \\
\cline { 2 - 6 } & Biosimilar 2 & 42 & 41 & $97.6 \%$ & Rettenbacher et al and \\
\hline \multirow{2}{*}{$\begin{array}{l}\text { Originator vs } \\
\text { pooled } \\
\text { biosimilars }\end{array}$} & \begin{tabular}{l} 
Originator \\
\cline { 2 - 6 }
\end{tabular} & 100 & 97 & $97.0 \%$ & Strowitzki et al \\
\hline
\end{tabular}

Notes: Biosimilar I: Bemfola $^{\circledR}$, Gedeon Richter UK Ltd, London, UK. Biosimilar 2: Ovaleap ${ }^{\circledR}$; CVC Capital Partners, Luxembourg.

Table 2 Mild-moderate and severe OHSS event rates

\begin{tabular}{|c|c|c|c|c|c|c|c|c|}
\hline \multirow[t]{2}{*}{ Analysis } & \multirow[t]{2}{*}{ Intervention } & \multicolumn{3}{|c|}{$\begin{array}{l}\text { Mild-moderate } \\
\text { OHSS }\end{array}$} & \multicolumn{3}{|c|}{$\begin{array}{l}\text { Severe } \\
\text { OHSS }\end{array}$} & \multirow[t]{2}{*}{ Reference } \\
\hline & & $\mathbf{n}$ & $\mathbf{N}$ & $\%$ & $\mathbf{n}$ & $\mathbf{N}$ & $\%$ & \\
\hline \multirow[t]{2}{*}{ Originator vs Biosimilar I } & Originator & 15 & 123 & 12.2 & 1 & 123 & 0.8 & \multirow{2}{*}{ Rettenbacher et al $2015^{14}$} \\
\hline & Biosimilar I & 53 & 249 & 21.3 & 2 & 249 & 0.8 & \\
\hline \multirow[t]{2}{*}{ Originator vs Biosimilar 2} & Originator & 3 & 146 & 2.1 & 1 & 146 & 0.7 & \multirow{2}{*}{ Strowitzki et al $2016^{15}$} \\
\hline & Biosimilar 2 & 6 & 153 & 3.9 & I & 153 & 0.7 & \\
\hline \multirow[t]{2}{*}{ Originator vs pooled biosimilars } & Originator & 18 & 269 & 6.7 & 2 & 269 & 0.7 & \multirow{2}{*}{ Rettenbacher et al and Strowitzki et al ${ }^{14,15}$} \\
\hline & Pooled biosimilars & 59 & 402 & 14.7 & 3 & 402 & 0.7 & \\
\hline
\end{tabular}

Notes: Biosimilar I: Bemfola $^{\circledR}$, Gedeon Richter UK Ltd, London, UK. Biosimilar 2: Ovaleap ${ }^{\circledR}$; CVC Capital Partners, Luxembourg.

Table 3 Daily dosing inputs for normal, hyper- and poor responders

\begin{tabular}{|c|c|c|c|c|c|c|}
\hline Analysis & Intervention & $\begin{array}{l}\text { Mean normal } \\
\text { responder dose }\end{array}$ & $\begin{array}{l}\text { Mean hyper- } \\
\text { responder dose }\end{array}$ & $\begin{array}{l}\text { Mean poor- } \\
\text { responder dose }\end{array}$ & $\begin{array}{l}\text { Duration } \\
\text { (days) }\end{array}$ & Reference \\
\hline \multirow{2}{*}{$\begin{array}{l}\text { Originator vs } \\
\text { Biosimilar I }\end{array}$} & Originator & I47 IU & I22 IU & I7I IU & 11 & \multirow{2}{*}{$\begin{array}{l}\text { Rettenbacher } \\
\text { et al } 2015^{14}\end{array}$} \\
\hline & Biosimilar I & I $47 \mathrm{IU}$ & 11910 & I $74 \mathrm{IU}$ & 11 & \\
\hline \multirow{2}{*}{$\begin{array}{l}\text { Originator vs } \\
\text { Biosimilar } 2\end{array}$} & Originator & I66 IU & $116 \mathrm{IU}$ & $216 \mathrm{IU}$ & 10 & \multirow{2}{*}{$\begin{array}{l}\text { Strowitzki et a } \\
2016^{15, a}\end{array}$} \\
\hline & Biosimilar 2 & I65 IU & $112 \mathrm{IU}$ & $218 \mathrm{IU}$ & 9 & \\
\hline
\end{tabular}

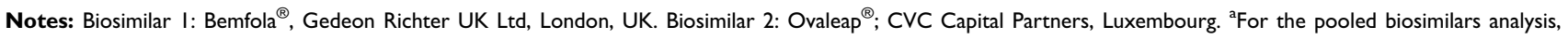
dosing for each arm was assumed to equal that from Strowitzki et al 2016.

\section{Assisted reproduction and birth costs}

Individual components and total costs estimated for each stage of the treatment cycle (preparation, oocyte retrieval, no oocyte retrieval, embryo transfer etc) are outlined in Table 4. In some instances, where the cost was dependent on the type of ART carried out (IVF or ICSI), the cost was weighted by the proportion of IVF $(24 \%)$ and ICSI $(76 \%)$ procedures carried out, according to the German IVF register $2016 .{ }^{1}$
It was assumed that the cost of "preparation" for an ART cycle was comprised of the cost of general treatment, the treatment plan and serological tests, as outlined in the Kassenärztliche Bundesvereinigung (KBV) (Table 4). ${ }^{28}$ Whilst patients also receive medications during preparation for ART, prior to the administration of gonadotropins for COS, it was assumed that there would be no differences in treatment between the 
Table 4 Unit cost: assisted reproduction and birth

\begin{tabular}{|c|c|c|}
\hline Cost component & Cost (EUR $€)$ & Reference \\
\hline \multicolumn{3}{|l|}{ Preparation } \\
\hline $\begin{array}{l}\text { General treatment, } \\
\text { treatment plan }\end{array}$ & 22.59 & \multirow{2}{*}{$\begin{array}{l}\text { Kassenärztliche } \\
\text { Bundesvereinigung } \\
\text { (KBV) }\end{array}$} \\
\hline $\begin{array}{l}\text { Serological tests } \\
\left(\mathrm{HIV}, \mathrm{Hb}_{\mathrm{c}}, \mathrm{HCV}, \mathrm{Hb}_{\mathrm{s}}\right)\end{array}$ & 50.60 & \\
\hline Total & 73.19 & Calculated \\
\hline \multicolumn{3}{|l|}{ Oocyte retrieval } \\
\hline $\begin{array}{l}\text { ICSI consultation for } \\
\text { couples }\end{array}$ & 8.63 & $\begin{array}{l}\text { Kassenärztliche } \\
\text { Bundesvereinigung } \\
(\mathrm{KBV})^{13}\end{array}$ \\
\hline $\begin{array}{l}\text { Outpatient surgery } \\
\text { visit, including post- } \\
\text { procedure } \\
\text { surveillance }\end{array}$ & 101.10 & $\begin{array}{l}\text { Kassenärztliche } \\
\text { Bundesvereinigung } \\
(\mathrm{KBV})^{13}\end{array}$ \\
\hline $\begin{array}{l}\text { Preparation of sperm } \\
\text { and processing } \\
\text { medium }\end{array}$ & 42.40 & $\begin{array}{l}\text { Kassenärztliche } \\
\text { Bundesvereinigung } \\
\text { (KBV), Federal } \\
\text { Association of } \\
\text { Centres for } \\
\text { Reproductive } \\
\text { Medicine } 11,13\end{array}$ \\
\hline $\begin{array}{l}\text { Ovum pick-up and } \\
\text { biopsy needle }\end{array}$ & 114.72 & $\begin{array}{l}\text { Kassenärztliche } \\
\text { Bundesvereinigung } \\
\text { (KBV), Federal } \\
\text { Association of } \\
\text { Centres for } \\
\text { Reproductive } \\
\text { Medicine } \\
13,28\end{array}$ \\
\hline $\begin{array}{l}\text { Anesthesia, including } \\
\text { monitoring }\end{array}$ & 119.17 & $\begin{array}{l}\text { Kassenärztliche } \\
\text { Bundesvereinigung } \\
(\mathrm{KBV})^{13}\end{array}$ \\
\hline Total & 463.95 & Calculated \\
\hline No oocyte retrieval & & \\
\hline $\begin{array}{l}\text { Discontinuation } \\
\text { before ovum pickup }\end{array}$ & 212.13 & $\begin{array}{l}\text { Kassenärztliche } \\
\text { Bundesvereinigung } \\
(\mathrm{KBV})^{13}\end{array}$ \\
\hline $\begin{array}{l}\text { ICSI consultation for } \\
\text { couples }\end{array}$ & 8.63 & $\begin{array}{l}\text { Kassenärztliche } \\
\text { Bundesvereinigung } \\
(\mathrm{KBV})^{13}\end{array}$ \\
\hline $\begin{array}{l}\text { Anesthesia, including } \\
\text { monitoring }\end{array}$ & 119.17 & $\begin{array}{l}\text { Kassenärztliche } \\
\text { Bundesvereinigung } \\
(\mathrm{KBV})^{13}\end{array}$ \\
\hline Total & 417.86 & Calculated \\
\hline
\end{tabular}

(Continued)
Table 4 (Continued).

\begin{tabular}{|c|c|c|}
\hline Cost component & Cost (EUR $€)$ & Reference \\
\hline \multicolumn{3}{|l|}{ Embryo transfer } \\
\hline $\begin{array}{l}\text { ICSI and embryo } \\
\text { transfer }\end{array}$ & $1,316.66$ & \multirow{2}{*}{$\begin{array}{l}\text { Kassenärztliche } \\
\text { Bundesvereinigung } \\
(\mathrm{KBV})^{13}\end{array}$} \\
\hline $\begin{array}{l}\text { IVF and embryo } \\
\text { transfer }\end{array}$ & 932.60 & \\
\hline Total (weighted) & $\mathrm{I}, 224.33$ & $\begin{array}{l}\text { Calculated (based on } \\
\text { proportion of IVF } \\
{[24 \%] / I C S I[76 \%] \text { ) }}\end{array}$ \\
\hline
\end{tabular}

\begin{tabular}{|l|l|l|}
\hline \multicolumn{2}{|l|}{ No embryo transfer } & \multicolumn{2}{l|}{} \\
\hline $\begin{array}{l}\text { ICSI, no embryo } \\
\text { transfer }\end{array}$ & $1,179.11$ & $\begin{array}{l}\text { Kassenärztliche } \\
\text { Bundesvereinigung } \\
(\text { KBV) }\end{array}$ \\
\hline $\begin{array}{l}\text { IVF, no embryo } \\
\text { transfer }\end{array}$ & 932.60 & $\left.\left.\left.\begin{array}{l}\text { Calculated (based on } \\
\text { proportion of IVF } \\
{[24 \%] / I C S I}\end{array}\right] 76 \%\right]\right)$ \\
\hline Total (weighted) & $1,119.85$ & \\
\hline
\end{tabular}

\section{Pregnancy/no pregnancy}

\begin{tabular}{|c|c|c|}
\hline $\begin{array}{l}\text { Blood test for beta } \\
\text { human chorionic } \\
\text { gonadotropin (preg- } \\
\text { nancy test) }\end{array}$ & 6.10 & $\begin{array}{l}\text { Kassenärztliche } \\
\text { Bundesvereinigung } \\
(\mathrm{KBV})^{13}\end{array}$ \\
\hline \multicolumn{3}{|l|}{ Live birth } \\
\hline Live birth & $3,686.12$ & $\begin{array}{l}\text { Fallpauschalen- } \\
\text { Katalog: Weighted } \\
\text { average of vaginal and } \\
\text { C-section births, }{ }^{14} \\
\text { Statistisches } \\
\text { Bundesamt }^{30}\end{array}$ \\
\hline \multicolumn{3}{|l|}{ Miscarriage } \\
\hline Miscarriage & 302.90 & $\begin{array}{l}\text { Kassenärztliche } \\
\text { Bundesvereinigung } \\
(\mathrm{KBV})^{13}\end{array}$ \\
\hline
\end{tabular}

Abbreviations: HIV, human immunodeficiency virus; $\mathrm{Hb}_{c}$, hepatitis $\mathrm{B}_{c}$ antigens; $\mathrm{HCV}$, hepatitis $\mathrm{C}$ virus; $\mathrm{Hb}_{\mathrm{s}}$, hepatitis $B$ surface antigen; ICER, incremental costeffectiveness ratio; ICSI, intracytoplasmic sperm injection; IVF, in-vitro fertilization.

Originator and biosimilars and, therefore, these costs were excluded from the analysis.

The cost of a live birth was assumed to be composed of the weighted average of vaginal and $\mathrm{C}$-section births from the Fallpauschalen-Katalog, based on a reported proportion of $30.5 \%$ C-section births in Germany in 2016 (Table 4). ${ }^{29,30}$ 
Table 5 Unit cost: mild to moderate and severe OHSS

\begin{tabular}{|c|c|c|}
\hline $\begin{array}{l}\text { Cost } \\
\text { component }\end{array}$ & $\begin{array}{l}\text { Cost } \\
(E U R €)\end{array}$ & Reference \\
\hline \multicolumn{3}{|c|}{ Mild-moderate OHSS } \\
\hline Hematology test $(x 2)$ & 0.25 & \multirow{5}{*}{$\begin{array}{l}\text { Kassenärztliche } \\
\text { Bundesvereinigung (KBV) }\end{array}$} \\
\hline Hematocrit test $(x 2)$ & 0.25 & \\
\hline Creatinine test $(x 2)$ & 8.45 & \\
\hline Electrolyte test $(x 2)$ & 1.00 & \\
\hline Hepatic test $(x 2)$ & 10.25 & \\
\hline Total & 20.20 & Calculated \\
\hline \multicolumn{3}{|l|}{ Severe OHSS } \\
\hline $\begin{array}{l}\text { Poisoning/toxic } \\
\text { effects of drugs }\end{array}$ & $1,900.75$ & $\begin{array}{l}\text { GKV- Spitzenverband, DRG } \\
\times 62 Z^{36}\end{array}$ \\
\hline
\end{tabular}

Abbreviation: OHSS, ovarian hyper-stimulation syndrome.

The cost of a miscarriage was assumed to equal the cost for dilation and curettage, including anesthesia (Table 4). ${ }^{13}$

\section{Adverse event costs}

The cost components of mild-moderate and severe OHSS are outlined in Table 5. To mirror real-world practice in Germany, the base case analysis did not include the cost of vitrification or cryopreservation (egg-freezing) as part of the cost of severe OHSS, which can lead to cycle interruption. This is due to complexities in reimbursement of this procedure in Germany, which is normally self-funded. ${ }^{31}$

\section{Drug costs}

The German cost of each strength of each intervention were obtained from Lauer-Fischer $\mathrm{GmbH}$ WEBAPO ${ }^{\circledR}$ InfoSystem, using the ATC-group G03GA class. ${ }^{6}$ In Germany, the cost of the Originator preparations is higher compared to the biosimilar preparations. The pharmacyselling price (including value added tax) was used for all gonadotropins (Table 6). The final cost of each treatment was calculated as a weighted cost of the proportion of normal, hyper- and poor responders and the cost of the combination of vials required to achieve the normal, pooror hyper responder dose (Table 7). The final total weighted

Table 6 Unit cost: gonadotropin vial

\begin{tabular}{|c|c|c|c|c|}
\hline Vial strength (FSH IU) & Originator (EUR $€$ ) & Biosimilar I (EUR €) & Biosimilar 2 (EUR $€$ ) & Reference \\
\hline FSH $900 \mathrm{IU}$ & $537.26^{\mathrm{a}}$ & - & $430.01^{a}$ & \multirow{6}{*}{$\begin{array}{l}\text { Lauer-Fischer GmbH } \\
\text { WEBAPO }\end{array}$} \\
\hline FSH 450 IU & $274.13^{\mathrm{a}}$ & 227.64 & $220.00^{a}$ & \\
\hline FSH $300 \mathrm{IU}$ & $|86.4|^{\mathrm{a}}$ & 151.78 & $150.00^{\mathrm{a}}$ & \\
\hline FSH 225 IU & - & 116.50 & - & \\
\hline FSH I50 IU & - & 78.83 & - & \\
\hline FSH 75 IU & 53.69 & 42.95 & - & \\
\hline
\end{tabular}

Notes: ${ }^{a}$ Multi-dose vial. Biosimilar I: Bemfola ${ }^{\circledR}$, Gedeon Richter UK Ltd, London, UK. Biosimilar 2: Ovaleap ${ }^{\circledR}$; CVC Capital Partners, Luxembourg.

Abbreviation: $\mathrm{FSH}$, follicle stimulating hormone.

Table 7 Gonadotropin costs for normal, poor and hyper-responders for one fresh cycle based on daily dosing

\begin{tabular}{|c|c|c|c|c|c|}
\hline Comparison & $\begin{array}{l}\text { Normal respon- } \\
\text { der cost (EUR } €)\end{array}$ & $\begin{array}{l}\text { Poor responder } \\
\text { cost (EUR } € \text { ) }\end{array}$ & $\begin{array}{l}\text { Hyper-responder } \\
\text { cost (EUR } € \text { ) }\end{array}$ & $\begin{array}{l}\text { Weighted cost } \\
(E U R €)^{a}\end{array}$ & $\begin{array}{l}\Delta \text { Cost Originator vs } \\
\text { biosimilar (EUR } € \text { ) }\end{array}$ \\
\hline \multicolumn{6}{|c|}{ Originator vs Biosimilar $I^{14}$} \\
\hline Originator & 972.46 & $\mathrm{I}, 128.2 \mathrm{I}$ & 811.39 & 994.19 & \multirow{2}{*}{42.12} \\
\hline Biosimilar I & 867.13 & I,28I.50 & 867.13 & 952.08 & \\
\hline \multicolumn{6}{|c|}{ Originator vs Biosimilar $2^{15, b}$} \\
\hline Originator & $1,074.52$ & I,348.65 & 811.39 & $1,114.06$ & \multirow{2}{*}{222.23} \\
\hline Biosimilar 2 & 860.02 & I,080.02 & 650.01 & 891.83 & \\
\hline
\end{tabular}

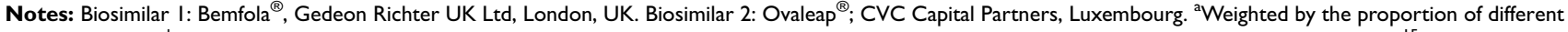
responder types; ${ }^{b}$ For the pooled biosimilar comparison, dosing for each arm and costs were assumed to equal those reported in the Biosimilar 2 trial. ${ }^{15}$ 
cost for the Originator and Biosimilar 1 was €994.19 and $€ 952.08$ respectively. The final average total weighted cost for Originator and Biosimilar 2 was $€ 1,114.06$ and $€ 891.83$ respectively. For the pooled biosimilars analysis, dosing and unit costs were conservatively assumed to equal dosing and costs for Biosimilar 2, as this was the lowest cost biosimilar.

\section{Sensitivity analysis}

One-way sensitivity analysis (OWSA) was conducted for all clinical and cost parameters by investigating the plausible upper and lower values from the reported outcomes. OWSA of cost input parameters was conducted by investigating outcomes around the upper and lower 25\% variance of input parameters. Scenario analyses on model outcomes were also conducted by changing the proportions of responder-types to $100 \%$ normal responders, $100 \%$ hyper-responders and $100 \%$ poor responders.

\section{Clinical expert validation}

Clinical and cost inputs, model structure and methodology were validated by a German clinical expert with extensive experience in assisted reproduction in Germany.

\section{Results}

The results of each analysis are outlined in Figure 2 and Tables 8-10. Results indicate that the Originator is associated with a higher rate of live birth and a lower cost per live birth compared to Biosimilar 1, Biosimilar 2 and pooled biosimilars.

\section{Clinical outcomes}

Following stimulation and one fresh embryo (non-frozen) transfer, the estimated live birth rate for the Originator was higher, compared to Biosimilar 1 (40.7\% vs $32.1 \%$ respectively), Biosimilar 2 (32.2\% vs $26.8 \%$ respectively) and the pooled biosimilars $(36.0 \%$ vs $30.1 \%$ respectively).

\section{Cost outcomes}

The average total costs for women treated with the Originator were estimated to be higher than those treated with biosimilars: the Originator versus Biosimilar 1 $(€ 4,272$ vs $€ 3,917)$, the Originator versus Biosimilar 2 ( $€ 4,053$ vs $€ 3,646)$, and the Originator versus pooled biosimilars $(€ 4,207$ vs $€ 3,777)$. For each comparison, cost breakdown (gonadotropin costs, adverse event costs, assisted reproduction costs and birth costs) were reported as the average cost for patients treated with the Originator, Biosimilar 1 and Biosimilar 2. The estimated gonadotropin costs account for a small proportion of total costs in all treatment arms (23.3-27.5\% for the Originator and $24.3 \%, 24.5 \%$ and $23.6 \%$ for the Biosimilar 1, Biosimilar 2 and pooled biosimilars respectively), compared to the sum of assisted reproduction and live birth costs.

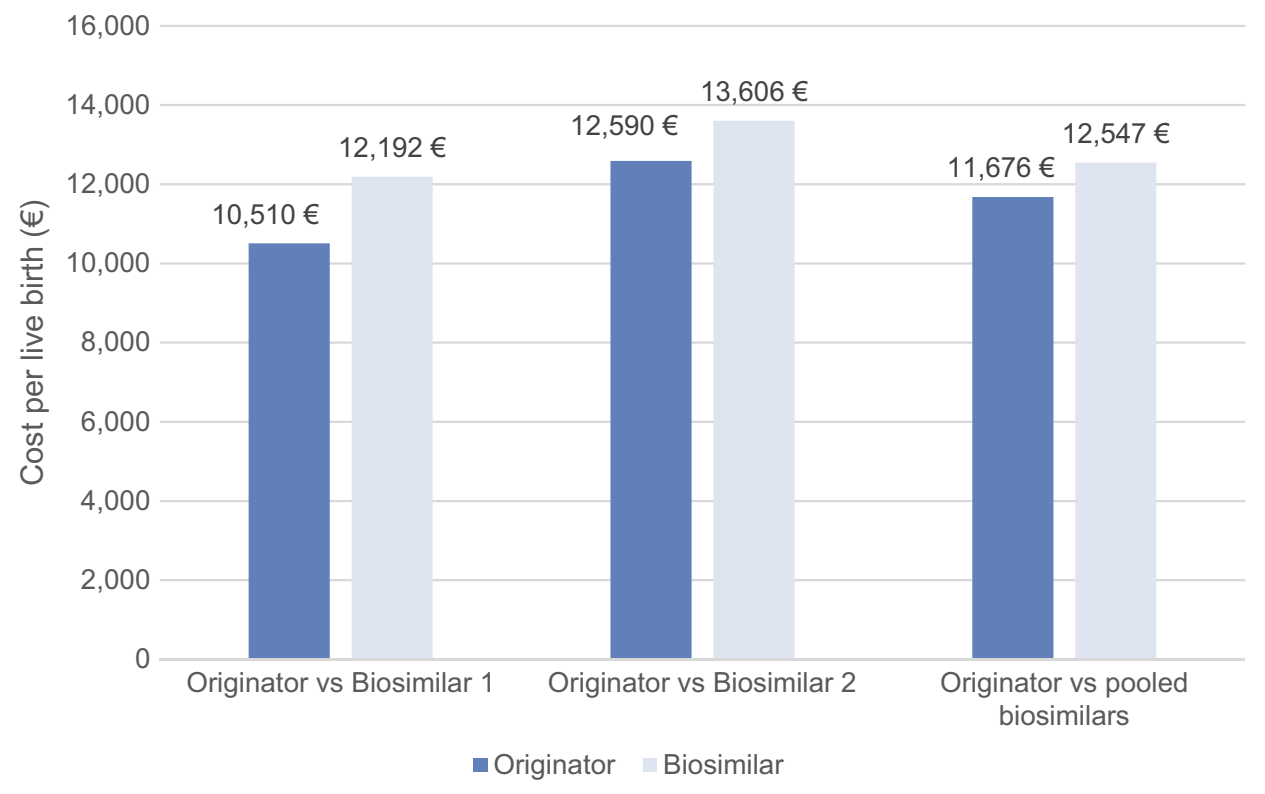

Figure 2 Cost per live birth for Originator versus Biosimilar I, Biosimilar 2 and pooled biosimilars.

Notes: Biosimilar I: Bemfola ${ }^{\circledR}$, Gedeon Richter UK Ltd, London, UK. Biosimilar 2: Ovaleap ${ }^{\circledR}$; CVC Capital Partners, Luxembourg. 
Table 8 Key clinical and cost-effectiveness results for Originator versus Biosimilar I

\begin{tabular}{|l|l|l|l|}
\hline & Originator & Biosimilar I & Difference \\
\hline Live birth rate & $40.7 \%$ & $32.1 \%$ & $8.5 \%$ \\
\hline Total costs $(€)^{\mathrm{a}}$ & 4,272 & 3,917 & 355 \\
\hline Gonadotropin costs $(€)$ & 994 & 952 & 42 \\
\hline Adverse event costs $(€)$ & 18 & 20 & -2 \\
\hline Assisted reproduction costs $(€)$ & 1,759 & 1,756 & 3 \\
\hline Birth costs $(€)$ & 1,501 & 1,189 & 312 \\
\hline Cost per live birth $(€)$ & 10,510 & $\mathbf{1 2 , 1 9 2}$ & \\
\hline ICER $(€)$ & $\mathbf{4 , 1 6 8}$ & \\
\hline
\end{tabular}

Notes: Biosimilar I: Bemfola ${ }^{\circledR}$, Gedeon Richter UK Ltd, London, UK. ${ }^{\mathrm{a}}$ Cost breakdowns were estimated as the average cost for women within each treatment arm.

Abbreviation: ICER, incremental cost-effectiveness ratio.

Table 9 Key clinical and cost-effectiveness results for Originator versus Biosimilar 2

\begin{tabular}{|c|c|c|c|}
\hline & Originator & Biosimilar 2 & Difference \\
\hline Live birth rate & $32.2 \%$ & $26.8 \%$ & $5.4 \%$ \\
\hline Total costs $(€)^{\mathrm{a}}$ & 4,053 & 3,646 & 407 \\
\hline Gonadotropin costs $(€)$ & $\mathrm{I}, \mathrm{II}$ & 892 & 222 \\
\hline Adverse event costs $(€)$ & 13 & 13 & 0 \\
\hline Assisted reproduction costs $(€)$ & $\mathrm{I}, 735$ & $|, 75|$ & -17 \\
\hline Birth costs $(€)$ & 1,191 & 990 & 201 \\
\hline Cost per live birth $(€)$ & 12,590 & 13,606 & \\
\hline ICER (€) & \multicolumn{2}{|c|}{7,540} & \\
\hline
\end{tabular}

Notes: Biosimilar 2: Ovaleap ${ }^{\circledR}$; CVC Capital Partners, Luxembourg. ${ }^{\mathrm{a} C o s t}$ breakdowns were estimated as the average cost for women within each treatment arm. Abbreviation: ICER, incremental cost-effectiveness ratio.

Table 10 Key clinical and cost-effectiveness results for Originator versus pooled biosimilars

\begin{tabular}{|l|l|l|l|}
\hline & Originator & Pooled biosimilars & Difference \\
\hline Live birth rate & $36.0 \%$ & $30.1 \%$ & $5.9 \%$ \\
\hline Total costs $(€)^{\mathrm{a}}$ & 4,207 & 3,777 & 430 \\
\hline Gonadotropin costs $(€)$ & 1,114 & 892 & 222 \\
\hline Adverse event costs $(€)$ & 15 & 17 & -2 \\
\hline Assisted reproduction costs $(€)$ & 1,746 & 1,754 & -8 \\
\hline Birth costs $(€)$ & 1,332 & 1,113 & 218 \\
\hline Cost per live birth $(€)$ & $\mathbf{1 1 , 6 7 6}$ & $\mathbf{1 2 , 5 4 7}$ & $\mathbf{7 , 2 5 6}$ \\
\hline ICER $(€)$ & & \\
\hline
\end{tabular}

Note: ${ }^{2}$ Cost breakdowns were estimated as the average cost for women within each treatment arm.

Abbreviation: ICER, incremental cost-effectiveness ratio. 


\section{Cost-effectiveness}

Results suggested that the estimated average cost per live birth for women treated with the Originator are lower than those treated with biosimilars: Originator versus Biosimilar 1 ( $€ 10,510$ vs $€ 12,192)$; Originator versus Biosimilar 2 ( $€ 12,590$ vs $€ 13,606)$, and Originator versus pooled biosimilars ( $€ 11,676$ vs $€ 12,547)$. The Originator was associated with an ICER of $€ 4,168, € 7,540$ and $€ 7,256$ versus Biosimilar 1, Biosimilar 2 and pooled biosimilars respectively.

\section{Sensitivity analysis}

OWSA for incremental live births of the Originator versus the biosimilar products, indicated that model results were most sensitive to the probability of pregnancy for all interventions. Other key drivers included the probability of embryo transfer and the probability of live birth for all interventions. Scenario analyses varying the proportions of responder-types had minimal impact on the overall results, suggesting the robustness of the model outcomes.

\section{Discussion}

This study investigated the cost and clinical outcomes of the Originator in comparison to Biosimilar 1, Biosimilar 2 and pooled biosimilars, from a German payer perspective. Biosimilar follitropin alfa preparations have demonstrated non-inferiority to their reference medicinal product, the Originator, in terms of oocyte retrieval. ${ }^{14,15}$ However, the impact on live birth rates and costs associated with the entire treatment have not yet been established. The results of this study indicated that in Germany the Originator is associated with a higher live birth rate and a lower cost per live birth compared to Biosimilar 1 and Biosimilar 2 (Figure 2).

Previous publications comparing the Originator to Biosimilar 1 and Biosimilar 2 have concluded comparability based on the number of oocytes retrieved, the primary endpoint recommended by the EMA). ${ }^{14,15,21}$ While there is no meaningful difference in the number of oocytes retrieved between originator and biosimilar products, no assessment of the qualitative difference and how this affects other outcomes, such as live birth, has been undertaken. The clinical end-point of this analysis was the rate of live birth, which, as stipulated by the participants at the Harbin Consensus Conference, is the preferred primary outcome for all clinical trials of treatment for infertility. ${ }^{32}$ Whilst comparing the number of oocytes may be an effective measure of in-vivo efficacy, the live birth rate is a more clinically meaningful measure to payers, patients and IVF specialists. ${ }^{2,16}$ In the biosimilars trials, live birth was not shown to be similar, although neither study was adequately powered to detect this outcome. However, in a post-hoc analysis of the Rettenbacher ${ }^{14}$ and Strowitzki ${ }^{15}$ trials, the data reported in the EMA public assessment were pooled and analysed using an additive logistic regression model and maximum likelihood estimate, showing live birth rates of $35.8 \%$ for Gonal-f versus $30.3 \%$ for the pooled biosimilars $(p=0.034){ }^{33}$ The incremental live birth rate for the Originator compared to Biosimilar 1, Biosimilar 2 and pooled biosimilars based on clinical trial results is $8.5 \%, 5.4 \%$ and $5.9 \%$, respectively. When translated into a potential real-world setting, where a cohort of 1,000 women undergo COS as a part of ART, treatment with the Originator may result in 59-85 more live births compared to biosimilars. The difference in the live birth rate may be explained by variations in the structural profiles and manufacturing processes of the biosimilar and reference product, potentially leading to variations in FSH receptor activation and thus biological activity, as investigated by Mastrangeli et $\mathrm{al}^{20}$.

Total costs, including gonadotropins but excluding other ART medications, for one fresh cycle of assisted reproduction (IVF/ICSI) were estimated to range between $€ 4,053-€ 4,272$ for the Originator and $€ 3,777-€ 3,646$ for biosimilars. These estimates are similar to a previous estimate by Rauprich et al, which estimated the cost of a standard IVF cycle in Germany, including medication, to be about $€ 3,000$ and an ICSI to be about $€ 3,600$ in $2008 .{ }^{3}$ Our findings suggested that gonadotropin costs may account for a smaller proportion of total costs compared to the combined cost of assisted reproduction and birth. In our model, total costs were largely driven by the average birth costs, which accounted for $29-35 \%$ of total costs.

It was found that the estimated average cost per live birth is lower for the Originator versus Biosimilar 1 $(€ 10,510$ versus $€ 12,192)$ and Biosimilar 2 (€12,590 versus $€ 13,606)$. An additional analysis using the pooled data from both biosimilar trials confirmed the individual analysis for the Originator versus Biosimilar 1 and Biosimilar 2 $(€ 11,676$ versus $€ 12,547)$. In our analysis, the ICER for the Originator was estimated to be $€ 4,168, € 7,540$ and $€ 7,256$ versus Biosimilar 1, Biosimilar 2 and the pooled biosimilars respectively.

The results from this study are in line with previous cost-effectiveness analyses of follitropin alfa products. Silverio et al (2015) estimated the cost-effectiveness of the Originator versus Biosimilar 1 over one treatment 
cycle in Portugal. The cost per live birth was estimated to be lower for the Originator in comparison with Biosimilar 1, at $€ 7,534.49$ versus $€ 9,205.31$, respectively. ${ }^{34}$ Gizzo et al (2016) estimated the cost-effectiveness of the Originator compared to Biosimilar 1 over two treatment cycles in Italy and Spain. The cost per live birth was also estimated to be lower for Originator in Italy and Spain, $€ 7,044$ and $€ 12,283$ respectively, compared to $€ 7,411$ and $€ 13,494$ for Biosimilar 1. ${ }^{23}$ Like the analysis presented in the current study, the analyses by Silverio et al (2015) and Gizzo et al (2016) were based on clinical evidence reported by Rettenbacher et $\mathrm{al}^{14}$. Gizzo et al (2016) acknowledged that Rettenbacher et $\mathrm{al}^{14}$ was not powered to demonstrate the live-birth rate, in addition to other study limitations, such as the use of data reported in the literature.

In another study, Gizzo et al (2018) estimated the costeffectiveness of the Originator compared to Biosimilar 2, based on live birth rates reported in Strowitzki et $\mathrm{al}^{15}$. Gizzo et al (2018) also reported a lower cost per live birth for the Originator versus the Biosimilar 2 in three countries (Germany $€ 8,135.04$ versus $€ 9,185.34$; Italy $€ 8,545.22$ versus $€ 9,733.37$; Spain $€ 14,859.53$ versus $€ 17,767.19) .{ }^{22}$ Similarly, Silverio et al (2016) estimated the cost-effectiveness of the Originator versus the Biosimilar 2 in Portugal, based on data from Strowitzki et $\mathrm{al}^{15}$. The authors found that in Portugal, the Originator was also associated with a lower cost per live birth (€9,391.67), compared to Biosimilar 2 (€10,977.42). ${ }^{35}$

A German study in 2008, estimated the cost per live birth to be approximately $€ 15,000{ }^{3}$ These costs are in line with the current study estimates for the cost per live birth, which were estimated to range between $€ 10,510-€ 12,590$ for the Originator and $€ 12,192, € 13,606$ and $€ 12,547$ for Biosimilar 1, Biosimilar 2 and pooled biosimilars respectively.

The authors of the current study acknowledge that the results obtained in this study are subject to some limitations, primarily related to a lack of clinical trial data or real-world evidence. This analysis compared the Originator to each biosimilar over one ART cycle rather than over multiple cycles, as often occurs in real-world practice. However, given that live birth rates decline for repeated cycles of ART, ${ }^{1}$ it was thought that in the absence of data to inform the probability of subsequent live birth rates, extrapolating the available evidence over successive cycles would be misleading. Related to this, due to a lack of data reported in the trials for Biosimilar $1^{14}$ and
Biosimilar $2,{ }^{15}$ it was not possible to account for the patients who discontinued or interrupted a cycle due to OHSS. To overcome this limitation, the authors assumed that these patients would be accounted for in the "no oocyte retrieval" of "no oocyte embryo transfer" states. Another limitation relates to the studies by Rettenbacher et $\mathrm{al}^{14}$ and Strowitzki et $\mathrm{al},{ }^{15}$ which were not designed or powered to assess live birth rates. Several of these limitations could be overcome by utilizing real-world evidence, mirroring efficacy in populations in countries of interest. As previously discussed, our current analysis is an exploratory one that is based on live-birth rates because this is a more valid endpoint for ART than the number of oocytes retrieved and is the only outcome that can be measured in terms of cost-effectiveness. In order for live birth rates to be considered as a primary outcome, a much larger sample size would be required, to achieve sufficient statistical power. However, in the absence of such publicly available data, implementing data as reported in the literature provides a measure of clinical efficacy and costeffectiveness.

\section{Conclusion}

Within the limitations of this study, our results suggest that in comparison to Biosimilar 1 and Biosimilar 2, the Originator is associated with a higher live birth rate and a lower cost per live birth in Germany. This analysis therefore could carry implications on the perception of "value for money" with lower-cost biosimilar follitropin alfa preparations. This study was based on the only efficacy and safety data currently available for the biosimilars. We acknowledge that further research is required to validate our results, with studies using real-world data and adequate power to detect the significance of these findings.

\section{Acknowledgment}

This study was financially supported by Merck KGaA.

\section{Author contributions}

WX, AL and EF were responsible for the creation of study documents, data analysis, data interpretation, and writing of the manuscript. All authors contributed to data analysis, drafting and revising the article, gave final approval of the version to be published, and agree to be accountable for all aspects of the work. 


\section{Disclosure}

WX, AL and EF are employees of IQVIA, London, UK and $\mathrm{CR}$ and $\mathrm{RP}$ are employees of Merck KGaA. KB has received honoraria from Merck. WX reports personal fees from MSD, Teva, and Ferring, during the conduct of the study; AL reports grants from Merck KGaA, during the conduct of the study; grants from Merck Inc, Ferring, and Teva, outside the submitted work; EF reports personal fees from Merck KGaA, during the conduct of this study; CR is a consultant for Merck. The authors report no other conflicts of interest in this work.

\section{References}

1. Blumenauer V, Czeromin U, Fehr D, et al. D.I.R-annual 2016 - the German IVF-registry. J Reproduktionsmed Endokrinol. 2017;14 (6):272-305

2. Berg Bringham K, Cadier B, Chevreul K. The diversity of regulation and public financing of IVF in Europe and its impact on utilization. Hum Reprod. 2013;28(3):666-675. doi:10.1093/humrep/des418

3. Rauprich O, Bockenheimer-Lucis G, Thorn P, Wendehorst C. Sollen Kinderwunschbehandlungen von den Krankenkassen finanziert werden? Ethische und rechtliche Aspekte [Shall ART treatments be publicly financed? Ethical and legal aspects]. In: Bockenheimer-Lucius G, Thorn P, Wendehorst C, editors. Umwege zum eigenen Kind: Ethische und rechtliche Herausforderungen an die Reproduktionsmedizin 30 Jahre nach Louise Brown [Detours to a Child of One's Own: Ethical and Legal Challenges of Reproductive Medicine 30 Years after Louise Brown]. Göttingen: Universitätsverlag Göttingen; 2008:31-47.

4. Pouwer AW, Farquhar C, Kremer JA. Long-acting FSH versus daily FSH for women undergoing assisted reproduction. Cochrane Database Syst Rev. 2015;14(7):CD009577.

5. Van Wely M, Kwan I, Burt AL, et al. Recombinant versus urinary gonadotrophin for ovarian stimulation in assisted reproductive technology cycles. Cochrane Database Syst Rev. 2011;16(2):CD005354.

6. Bals-Pratsch M, Bühler K, Krüssel J, Wendelken M, Dahncke W, Kupka MS. Extended analyses of the German IVF registry (D.I.R): andrological aspects, medical economical assumptions related to the shift from IVF to ICSI and stimulation with gonadotropins. J Reproduktionsmed Endokrinol. 2010;7(1):40-44.

7. Fragoulakis V, Pescott CP, Smeenk JM, et al. Economic evaluation of three frequently used gonadotrophins in assisted reproduction techniques in the management of infertility in the Netherlands. Appl Health Econ Health Policy. 2016;14(6):719-727. doi:10.1007/s40258-016-0259-9

8. Katzorke T, Verhoeven H, Blechschmidt J, Kohler M, Kelly E. Follitropin-alfa ovarian stimulation during assisted reproduction treatment: a national collaborative study. Reprod Biomed Online. 2001;3(2):98-103.

9. Lass A, McVeigh E. Routine use of r-FSH follitropin alfa filled-bymass for follicular development for IVF: a large multicentre observational study in the UK. Reprod Biomed Online. 2004;9(6):604-610.

10. Lehert P, Schertz JC, Ezcurra D. Recombinant human follicle-stimulating hormone produces more oocytes with a lower total dose per cycle in assisted reproductive technologies compared with highly purified human menopausal gonadotrophin: a meta-analysis. Reprod Biol Endocrinol. 2010;8:112. doi:10.1186/1477-7827-8-112

11. Nawroth F, Tandler-Schneider A, Bilger W. Multicenter, noninterventional, post-marketing surveillance study to evaluate dosing of recombinant human follicle-stimulating hormone using the redesigned follitropin alfa pen in women undergoing ovulation induction. Drug Healthc Patient Saf. 2015;7:63-68. doi:10.2147/ DHPS.S76693
12. Trew GH, Brown AP, Gillard S, et al. In vitro fertilisation with recombinant follicle stimulating hormone requires less IU usage compared with highly purified human menopausal gonadotrophin: results from a European retrospective observational chart review. Reprod Biol Endocrinol. 2010;8:137. doi:10.1186/1477-7827-8-137

13. Weiss N. Gonadotrophin products: empowering patients to choose the product that meets their needs. Reprod Biomed Online. 2007;15 (1):31-37.

14. Rettenbacher M, Andersen AN, Garcia-Velasco JA, et al. A multicentre phase 3 study comparing efficacy and safety of Bemfola ${ }^{\circledR}$ versus Gonal- $\mathrm{f}^{\circledR}$ in women undergoing ovarian stimulation for IVF. Reprod Biomed Online. 2015;30(5):504-513. doi:10.1016/j. rbmo.2015.01.005

15. Strowitzki T, Kuczynski W, Mueller A, Bias P. Randomized, activecontrolled, comparative phase 3 efficacy and safety equivalence trial of Ovaleap $^{\mathbb{R}}$ (recombinant human follicle-stimulating hormone) in infertile women using assisted reproduction technology (ART). Reprod Biol Endocrinol. 2016;14:1. doi:10.1186/s12958-015-0135-8

16. Trappe H. Assisted reproductive technologies in Germany: a review of the current situation. In: Kreyenfeld M, Konietzka D, editors. Childlessness in Europe: Contexts, Causes, and Consequences. Cham: Springer International Publishing; 2017:269-288.

17. European Medicines Agency. Biosimilars in the EU; information guide for healthcare professionals; 2017. Available from: https:// www.ema.europa.eu/documents/leaflet/biosimilars-eu-informationguide-healthcare-professionals_en.pdf. Accessed January 30, 2019.

18. Bassett RM, Driebergen R. Continued improvements in the quality and consistency of follitropin alfa, recombinant human FSH. Reprod Biomed Online. 2005;10(2):169-177.

19. Hugues JN, Durnerin IC. Gonadotrophins-filled-by-mass versus filled-by-bioassay. Reprod Biomed Online. 2005;10(Suppl 3):11-17.

20. Mastrangeli R, Satwekar A, Cutillo F, Ciampolillo C, Palinsky W, Longobardi S. In-vivo biological activity and glycosylation analysis of a biosimilar recombinant human follicle-stimulating hormone product (Bemfola) compared with its reference medicinal product (GONAL-f). PLoS One. 2017;12(9):e1084139. doi:10.1371/journal.pone.0184139

21. Committee for Medicinal Products for Human Use (CHMP). Guideline on non-clinical and clinical development of similar biological medicinal products containing recombinant human follicle stimulating hormone (r-hFSH). European Medicines Agency (EMA); 2013. Available from: https://www.ema.europa.eu/documents/scienti fic-guideline/guideline-non-clinical-clinical-development-similarbiological-medicinal-products-containing_en.pdf. Accessed January $30,2019$.

22. Gizzo S, Ferrando M, Lispi M, Ripellino C, Cataldo N, Buhler K. A cost-effectiveness modelling evaluation comparing a biosimilar follitropin alfa preparation with its reference product for live-birth outcome in Germany, Italy and Spain. J Med Econ. 2018;21(11)10961101.

23. Gizzo S, Garcia-Velasco JA, Heiman F, Ripellino C, Buhler K. A cost-effectiveness evaluation comparing originator follitropin alfa to the biosimilar for the treatment of infertility. Int $J$ Womens Health. 2016;8:683-689. doi:10.2147/IJWH.S118687

24. Grynberg M, Murphy C, Dore C, et al. A cost-effectiveness analysis comparing the originator follitropin alfa to its biosimilars in patients undergoing a medically assisted reproduction program from a French perspective. J Med Econ. 2019;22:108-115. doi:10.1080/13696 998.2018.1551226

25. La Marca A, Sunkara SK. Individualization of controlled ovarian stimulation in IVF using ovarian reserve markers: from theory to practice. Hum Reprod Update. 2014;20(1):124-140. doi:10.1093/humupd/dmt037

26. Ferraretti AP, La Marca A, Fauser BC, et al. ESHRE consensus on the definition of 'poor response' to ovarian stimulation for in vitro fertilization: the Bologna criteria. Hum Reprod. 2011;26 (7):1616-1624. doi:10.1093/humrep/der092 
27. Sunkara SK, Rittenberg V, Raine-Fenning N, Bhattacharya S, Zamora J, Coomarasamy A. Association between the number of eggs and live birth in IVF treatment: an analysis of 400,135 treatment cycles. Hum Reprod. 2011;26(7):1768-1774. doi:10.1093/humrep/der106

28. Bundesverband Reproduktionsmedizinischer Zentren Deutschlands e. V. (BRZ). Costs for biopsy needle, sperm processing medium. Available from: http://www.repromed.de/. Accessed January 30, 2018.

29. Lauer-Fischer GmbH WEBAPO ${ }^{\circledR}$ InfoSystem. ATC-group G03GA [updated August 1, 2017]. Available from: https://www.cgm.com/ lauer-fischer/index.de.jsp. Accessed August 1, 2017.

30. Geschäftsverkehr(GKV)-Verband der Privaten Krankenversicherung e.V. and Deutsche Krankenhausgesellschaft; 2017. Available from: http://www.dkgev.de/media/file/27711. Vereinbarung Bundesbasisfallwert_2017. Accessed January 30, 2018.

31. Rauprich O, Berns E, Vollman J. Who should pay for assisted reproductive techniques? Answers from patients, professionals and the general public in Germany. Hum Reprod. 2010;25(5):1225-1233. doi:10.1093/humrep/deq056

32. Legro RS, Wu X, Barnhart KT, Farquhar C, Fauser BC, Mol B. Improving the reporting of clinical trials of infertility treatments (IMPRINT): modifying the CONSORT statementdaggerdouble dagger. Hum Reprod. 2014;29(10):2075-2082. doi:10.1093/humrep/deu218
33. Pasch RRC, D’Hoghe T, Longobardi S. Live Birth Rate (LBR), Ongoing Pregnancy Rate (OPR) And Ovarian Hyperstimulation Syndrome (OHSS) risk with originator versus biosimilar recombinant follitropin alfa: a pooled analysis of clinical trial data. PMU40. ISPOR Europe; November 10-14; 2018; Barcelona, Spain.

34. Silverio N, Batista AR, Sequeira L. Biosimilars, are they really cost saving? The case of recombinant human follicle stimulating hormone in Portugal. Value Health. 2015;18(7):A736. doi:10.1016/j. jval.2015.09.2820

35. Silverio N, Botica F, Batista A. Cost-effectiveness of recombinant human follicle stimulating hormone biosimilars in Portugal Value Health. 2016;19(7):A402-A403. doi:10.1016/j.jval.2016. 09.323

36. GKV-Spitzenverband. GKV-Verband der Privaten Kranken versicherung e.V. and Deutsche Krankenhausgesellschaft; 2017. Available from: http://www.dkgev.de/media/file/27711. Vereinbarung_Bundesbasisfallwert_2017. Accessed January 30, 2018.
International Journal of Women's Health

\section{Publish your work in this journal}

The International Journal of Women's Health is an international, peerreviewed open-access journal publishing original research, reports, editorials, reviews and commentaries on all aspects of women's healthcare including gynecology, obstetrics, and breast cancer. The manuscript management system is completely online and includes a very quick and fair peer-review system, which is all easy to use. Visit http://www.dovepress.com/testimonials.php to read real quotes from published authors. 